\title{
Resection of spinous processes can cause spinal cord injury in patient with ossification of the posterior longitudinal ligament in the thoracic spine
}

\author{
K Nakanishi, N Tanaka, N Kamei, T Hiramatsu, S Ujigo, N Sumiyoshi, T Rikita, A Takazawa and M Ochi
}

Study design: Case report.

Objective: To report intraoperative spinal cord injury by resection of spinous processes in a 73-year-old man with ossification of the posterior longitudinal ligament (OPLL) in the thoracic spine.

Methods: A 73-year-old man presented with cervicothoracic OPLL with bilateral numbness and clumsiness of his hand, weakness of his lower extremities and severe gait disturbance. His Japanese Orthopaedic Association (JOA) score was 7.5 out of 17. Cervical laminoplasty (C2-6), cervicothoracic laminectomy (C7-T10) and posterior fusion (C7-T10) were performed in the prone position with electrophysiologic monitoring of the spinal cord-evoked potentials (SCEPs).

Results: The spinal processes with supra- and interspinous ligaments between C7 and T10 were resected. After resection, the amplitude of SCEP waveforms decreased rapidly to $<10 \%$ of control levels. Laminectomy was performed, and, after 80 min of SCEP deterioration, an instrumented fusion with correction for kyphosis was completed. The SCEP amplitude recovered gradually. Immediately after surgery, the patient suffered severe motor loss in both lower limbs. His neurological recovery progressed gradually from 2 days after surgery, and he was able to walk at 3 months after surgery. At 6 years after surgery, the JOA score was 11 .

Conclusion: Our results suggest that intraoperative spinal cord injury can occur before posterior decompression by resection of spinal processes with supra- and interspinous ligaments. The timing of the instrumented stabilization using a temporary rod is important and should be considered immediately after posterior exposure of the spine.

Spinal Cord (2014) 52, S19-S21; doi:10.1038/sc.2014.151

\section{INTRODUCTION}

In treatment for thoracic ossification of the posterior longitudinal ligament (OPLL), postoperative paraplegia is a major risk. ${ }^{1,2}$ To avoid the postoperative paraplegia, posterior decompression with instrumented fusion (PDF) has been reported. ${ }^{3}$ However, postoperative paraplegia remains a risk and the causes are controversial. We present a case of cervicothoracic OPLL suggesting that electrophysiologic monitoring can be used to document the occurrence of paralysis with resection of spinous processes.

\section{CASE REPORT}

A 73-year-old man presented with cervicothoracic OPLL, a Japanese Orthopaedic Association (JOA) score for cervical myelopathy of 7.5 (17 points is full points) and JOA score for thoracic myelopathy of 5.5 (11 points is full points). The patient complained of bilateral numbness, clumsiness of his hands, bilateral motor weakness of his lower extremities (manual muscle testing: MMT at grade 4 out of 5), hypoesthesia in both lower limbs (Grade 1-5 out of 10) and inability to walk without a handrail. Reconstructed images from a computerized tomography (CT) myelogram showed extensive segmental OPLL, in particular at C4-5 (Figure 1a), T2-3 and T7-8 levels (Figure 1b). Thoracic MRI showed severe compression of the spinal cord at T2-3 and T7-8 (Figure 1c). The kyphotic Cobb's angle between the upper endplate of the $\mathrm{C} 7$ and the lower endplate of the $\mathrm{T} 10$ was $37^{\circ}$ in the sitting position. Posterior decompression at $\mathrm{C} 2-\mathrm{T} 10$ and posterior fusion at C7-T10 with instrumentation was conducted in the prone position. The patient was placed on a Hall frame with the skull fixed in a Mayfield three-point fixator and the cervical spine was positioned parallel to the floor. We were unable to detect motor evoked potentials following transcranial electric stimulation (TcMEPs), which suggested a severe corticospinal conduction block caused by the thoracic OPLL. Therefore, spinal cord-evoked potentials (SCEPs) following spinal cord electric stimulations were recorded. We applied catheter electrodes to the C2 and T10 subarachnoid space and stimulated the spinal cord via the dorsal dura at the C2 level with $0.2 \mathrm{~ms}$ square wave pulses of $10 \mathrm{~mA}$ constant current, and we were able to monitor SCEPs at the T10 level (Figure 2). ${ }^{4}$ The amplitude of the earliest negative waveform in the SCEPs was $17.6 \mu \mathrm{V}$ (control).

Cervical decompression was accomplished by a C2-C6 open door laminoplasty. Transpedicular screws were inserted at C7-T2 and T8-T10 bilaterally. The spinal processes with supra- and interspinous ligaments between $\mathrm{C} 7$ and $\mathrm{T} 10$ were resected. After resection, the amplitude of SCEP waveforms decreased rapidly to $<10 \%$ of control levels. Laminectomy between C7 and T10 was performed, and, after 
a

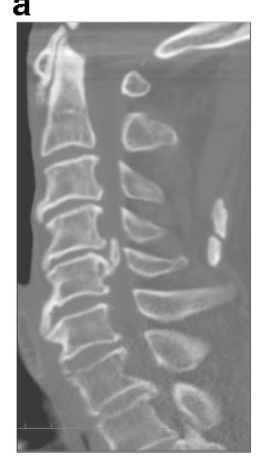

b

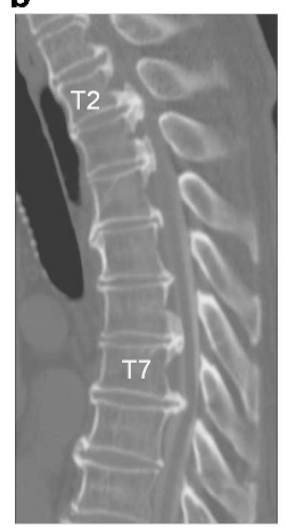

c

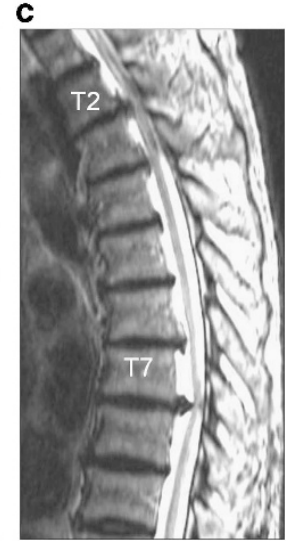

Figure 1 Reconstructed images from a CT myelogram in the cervical (a) and thoracic (b) spine showing extensive segmental OPLL at C4-5, T2-3 and T7-8 levels. (c) Thoracic magnetic resonance imaging showed severe compression to the spinal cord at T2-3 and T7-8.

80 min of SCEP deterioration, PDF with correction for kyphosis between C7 and T10 was completed using a spinal rod system. The SCEPs amplitude recovered gradually until the end of surgery.

Immediately after surgery, although improvements of numbness and clumsiness in the hands were observed, the patient suffered severe lower limb motor loss (MMT at Grade 0) and lower extremity hypoesthesia (Grade 1-3 out of 10). The kyphotic Cobb's angle was $31^{\circ}$ after surgery. Two days after surgery, we detected muscle contraction in the patient's lower limbs (MMT at Grade 1). His neurological recovery progressed gradually, and the patient could walk with a handrail at 3 months after surgery. At 6 years after surgery, the MMT in both lower limbs was recovered to Grade 5 and hypoesthesia was at Grade 8. Although he had frequent micturition, he was able to eat with chopsticks and his JOA score for cervical and thoracic myelopathy was 11 and 6.5, respectively.

\section{DISCUSSION}

We present a case of OPLL that showed a decrease of SCEP waveforms immediately after resection of spinous processes with supra- and interspinous ligaments. Tamaki et al. ${ }^{4}$ reported that six patients experienced postoperative neurologic complications, and that, in these patients, the peak amplitudes of the SCEPs diminished to less than $50 \%$ of the control values. Recently, Kobayashi et al. ${ }^{5}$ recommended the designation of an alarm point at a $70 \%$ decrease in TcMEPs amplitude for routine spinal cord monitoring. In accordance with those reports, we usually conduct spinal surgery using TcMEPs with an alarm point of less than $30 \%$ of the amplitude of the control values. When the TcMEPs could not be recorded because the myeopathy was severe, we perform the spinal cord monitoring using SCEPs. In the present case, the TcMEPs could not be detected and SCEPs were monitored. The amplitudes of the SCEPs diminished to less than $10 \%$ of the control values immediately after resection of the spinous process, which was lower than that in both previous reports, suggesting that the spinal cord function deteriorated during that period of time.

Progression of kyphosis is an important factor in paraplegia after posterior decompression for thoracic OPLL, and stabilization using a temporary rod is recommended. ${ }^{6}$ Matsuyama et al. ${ }^{7}$ reported posterior displacement of the spinal cord after laminectomy and concluded that destruction of the posterior spinal structures, such as

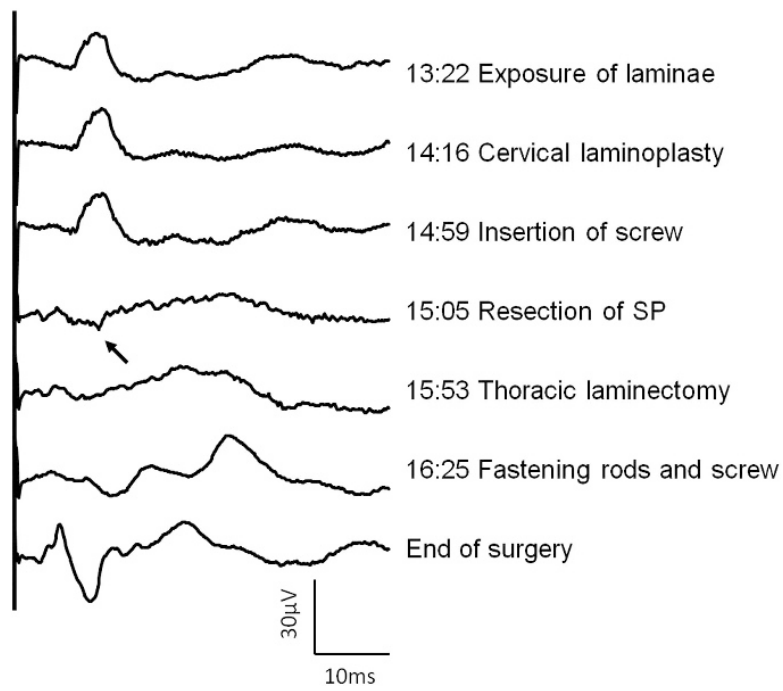

Figure 2 Waveforms from SCEP monitoring. The shapes of waveform varied after resection of the spinous process (SP) with supra- and interspinous ligaments between C7 and T10. A laminectomy between C7 and T10 was performed, after which, the amplitude of SCEP waveform decreased rapidly. After $80 \mathrm{~min}$, instrumented fusion (PDF) was completed using a spinal rod system. Then, the SCEP amplitude gradually recovered until the end of surgery. The point of change in waveform is indicated by an arrow.

occurs during a laminectomy procedure, may increase spinal kyphosis, leading to the spinal cord deterioration, in the beak type of thoracic OPLL. CT myelogram demonstrated a beak-type OPLL at the T7-8 level in the present case. Therefore, the resection of spinal processes can damage posterior spinal structures, increase kyphosis and lead to spinal cord compression by the beak-type OPLL.

In patients with thoracic OPLL, the spinal column shows some mobility at the cord compression level in spite of the presence of massive heterotopic vertebral ossification. ${ }^{8} \mathrm{~A}$ rapid decline of TcMEPs amplitude, even after placing a patient in a prone position, also has been reported. ${ }^{9}$ Therefore, the range of temporary stabilization should be wide enough to cover the cord compression site, as well as the physiological kyphotic region of the thoracic spine to avoid kyphotic progression. Moreover, our observations suggest that the timing of the instrumented stabilization is important and should be considered immediately after posterior exposure of spine.

\section{CONFLICT OF INTEREST}

The authors declare no conflict of interest.

\section{ACKNOWLEDGEMENTS}

This work was supported by the Grants-in-Aid for Scientific Research (Scientific Research C, Research Project Number: 24592198) from Japan Society for the Promotion of Science, Ministry of Health, Labour and Welfare.

1 Matsumoto M, Chiba K, Toyama Y, Takeshita K, Seichi A, Nakamura K et al. Surgical results and related factors for ossification of posterior longitudinal ligament of the thoracic spine: a multi-institutional retrospective study. Spine (Phila Pa 1976) 2008; 33: 1034-1041.

2 Yamazaki M, Koda M, Okawa A, Aiba A. Transient paraparesis after laminectomy for thoracic ossification of the posterior longitudinal ligament and ossification of the ligamentum flavum. Spinal Cord 2006; 44: 130-134. 
3 Nakanishi K, Tanaka N, Nishikawa K, Fujimoto Y, Ochi M. Positive effect of posterior instrumentation after surgical posterior decompression for extensive cervicothoracic ossification of the posterior longitudinal ligament. Spine 2005; 30: E382-E386.

4 Tamaki T, Noguchi T, Takano H, Tsuji H, Nakagawa T, Imai K et al. Spinal cord monitoring as a clinical utilization of the spinal evoked potential. Clin Orthop Relat Res 1984; 184: 58-64.

5 Kobayashi S, Matsuyama Y, Shinomiya K, Kawabata S, Ando M, Kanchiku T et al. A new alarm point of transcranial electrical stimulation motor evoked potentials for intraoperative spinal cord monitoring: a prospective multicenter study from the Spinal Cord Monitoring Working Group of the Japanese Society for Spine Surgery and Related Research. J Neurosurg Spine 2014; 20: 102-107.

6 Matsuyama Y, Yoshihara H, Tsuji T, Sakai Y, Yukawa Y, Nakamura H et al. Surgical outcome of ossification of the posterior longitudinal ligament (OPLL) of the thoracic spine: implication of the type of ossification and surgical options. J Spinal Disord Tech 2005; 18: 492-497; discussion 498.

7 Matsuyama Y, Sakai Y, Katayama Y, Imagama S, Ito Z, Wakao N et al. Indirect posterior decompression with corrective fusion for ossification of the posterior longitudinal ligament of the thoracic spine: is it possible to predict the surgical results? Eur Spine J 2009; 18: 943-948.

8 Yamazaki M, Okawa A, Fujiyoshi T, Furuya T, Koda M. Posterior decompression with instrumented fusion for thoracic myelopathy caused by ossification of the posterior longitudinal ligament. Eur Spine J 2010; 19: 691-698.

9 Ito Z, Imagama S, Ando K, Hirano K, Tauchi R, Muramoto A et al. The utility of $\mathrm{Br}(\mathrm{E})-\mathrm{MsEP}$ in surgeries for thoracic ossification of posterior longitudinal ligament. J Funct Diag Spinal Cord (in Japanese) 2012; 34: 175-181. 